\title{
Managing nutritional programmes in developing countries
}

\author{
R. Sheikholeslam, ${ }^{1}$ Z. Abdollahi ${ }^{2}$ and F.N. Haghighi ${ }^{2}$
}

\begin{abstract}
SUMMARY Improving community nutrition in developing countries requires a detailed epidemiological picture of the prevalent nutritional problems in different regions and age groups. This makes it possible to identify priorities, sensitize policy-makers, establish political commitment and design appropriate community programmes for income generation and education for the best use of food resources. Experiences acquired from community-based nutritional programmes show that ownership of a programme by the community and using a tailor-made approach are essential factors in the successful implementation of programmes. A multifaceted approach is needed, involving a range of sectors-agriculture, commerce, education and healthand commitment at all levels from government to communities and individuals.
\end{abstract}

\section{Introduction}

Current evidence suggests that developing countries are experiencing a transition phase in the epidemiology of nutritional disorders, and that most countries of the Eastern Mediterranean Region are entering this phase rapidly. In developing countries, there is a high prevalence of undernutrition (protein-energy malnutrition, and micronutrient deficiencies), which is most critical in the fetal and neonatal stages, as well as the under 5-year-old age group. On the other hand, with increasing urbanization in these countries, food consumption patterns are changing, physical activity is decreasing and metabolic disorders such as obesity, cardiovascular diseases, diabetes and cancer are also rising. It is not unusual in developing countries to see obese mothers who are caring for malnourished children. This shows that these countries face a bilateral problem, with a need both for decreasing malnutrition and micronutrient deficiencies, and for combating overnutrition by improving food consumption pat- terns and modifying lifestyles. In either case, the approach to planning and implementation of intervention programmes to improve nutrition are the same. This paper reviews strategies for managing nutritional programmes with examples of successful schemes from around the world.

\section{Effective planning}

Like other health programmes, the management of nutritional programmes should follow 3 consecutive stages: planning, implementation and evaluation. In the planning phase, 5 activities should be carried out: recognizing the situation; identifying problems and priorities; identifying goals; examining obstacles and difficulties; and devising a comprehensive action plan. Thus identifying and bringing together all the organizations and individuals who will play a part in the programme, to gain their cooperation and determine the goals of the programme, will be followed by a situation analysis to provide a general picture of the

${ }^{1}$ Director General, ${ }^{2}$ Department of Nutrition, Ministry of Health and Medical Education, Tehran, Islamic Republic of Iran.

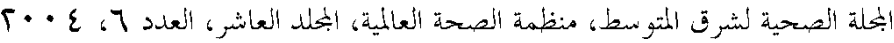


types and etiology of nutritional problems in the region.

Nutritional problems need to be tackled from a variety of angles. Holding brainstorming meetings and advocacy workshops with a whole range of sectors, including agriculture, commerce, education and health, as well as the community, is a effective method of analysing the problems in a region and promoting responsibility for solving them. Experiences of different countries have shown that if all members of communities participate in the problem-solving process, acquiring their cooperation in planned interventions is easier. After gathering the needed data and analysing the reasons for nutritional problems, the appropriate interventions, which are usually multi-faceted, should be designed and implemented. The selected approach may not be confined to the health sector and other sectors may have an even more important role to play.

\section{Communication for behavioural change}

Communication for behavioural change $(\mathrm{CBC})$ is one of the main strategies in improving community nutrition for increasing peoples' knowledge and motivation for change and is used for achieving different nutritional goals, such as improving complementary nutrition methods and increasing the acceptance of food supplements and the consumption of fortified foods. Women, especially mothers, are the key target group for modifying food habits for the health of both themselves and their children, and this is especially important in rural areas where mothers experience hard physical labour, multiple pregnancies and inadequate diets. Communication strategies have to include fathers too. In many areas, due to cultural reasons fathers have a higher share of the household's food in terms of quality and quality than other family members, especially women. In such cases, educational programmes for men are needed that focus on the nutritional needs of women and children.

Evaluation of CBC programmes on a large scale, especially those aiming at mothers and others who care for children, has shown they were effective in improving the nutritional status of children. One CBC programme, the Heart Model, has been implemented in Viet Nam, Bangladesh and Haiti [1]. It was part of a comprehensive intervention programme for growth monitoring, iron and vitamin A supplementation, de-worming and infection treatment. In this approach, mothers participated in educational sessions held in their own homes or visited rehabilitation centres for 2 weeks and were trained in preparing nutritious meals for their malnourished children using locally available foods. The programme has been successful in preventing mild to moderate malnutrition among children in Haiti and Viet Nam.

In the Islamic Republic of Iran, nutrition education was first implemented in 1995, in a rural area of Chahar-Mahal and Bakhtyari province [2]. In this programme, mothers who had children under 2 years old were educated in their homes using cooking demonstrations by community health workers and trained volunteer rural grandmothers. Raw materials for preparing baby foods were provided by mothers and the cooked meals were distributed among their children in health houses or the mother's home. The success of this programme in improving the nutritional status of rural children lead to its implementation across the whole health network of the country. Experiences of this programme have 
shown that practical nutrition education for mothers also provides opportunities for education about other issues such as growth monitoring, breastfeeding, nutrition in diarrhoea and other diseases.

\section{Promoting intra- and inter- sectoral communication}

Intra- and inter-sectoral communication is another key aspect of managing nutrition programmes. In the inter-sectoral approach, all health programmes affect the nutritional status of the community in some way. For example, control of diarrhoeal diseases and acute respiratory infections, healthy mother programmes, family planning, environmental modifications, vaccination, food safety programmes and controlling infectious and parasitic diseases are measures that should be done in parallel with nutritional services for improving the nutritional status of the community. It has become clear that there is a direct relation between the quality of drinking water supplies and the nutritional status of children, as contaminated water supplies increase the prevalence of diarrhoea and diarrhoea leads to nutritional deficiencies [3]. However, improving the quality of water supplies does not necessarily reduce the prevalence of malnutrition among children. In this same way, nutrition education alone does not improve nutritional status.

\section{Investing in women's literacy and education}

Experiences of different countries are that investing in literacy and health education, family planning and improving the quality of women's life has resulted in a sustainable improvement in the nutritional status of communities.
It has been shown that the mother's education has an even more positive effect on children's growth than the economic situation of the family. A study by the United Nations Children's Fund (UNICEF) of 23 nutritional projects in South-East Asia showed that the literacy of mothers is an essential factor in children's growth and improves nutritional status of children more rapidly than other interventional factors [4]. In an intervention plan to reduce malnutrition among children in rural areas of 3 provinces of the Islamic Republic of Iran over 3 years, a literacy programme for rural women resulted in a $10 \%$ increase in the number of literate women $(3 \%$ increase in the total number of literate people nationally). This was thought to have played an important part in reducing malnutrition measures among children in the region before and after the intervention [5].

Education about nutrition is another key factor in mothers' ability to provide appropriate care of family members. In the Iranian programme mentioned earlier, nutritional messages based on women's daily needs were included in literacy classes to reinforce the effect of the literacy education and increase the women's motivation for participating in classes [5]. Several studies have shown that education of mothers about nutrition not only decreases the proportion of underweight children, but also decreases child mortality [5-7]. It also has positive long-term effects on the children's future development.

\section{Integrating nutritional and health interventions}

In view of the multidimensional nature of nutritional problems, programmes and activities that do not directly relate to nutrition also need to be considered in designing in-

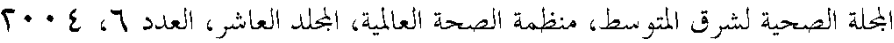


terventions for improving the nutritional status of communities. These include providing safe drinking water, modifying the environment, family planning, integrated management of childhood illnesses and other mother and child health programmes, modifying lifestyles and increasing physical activity. In most nutritional projects, in different settings, these measures have been included in nutritional interventions [8].

An example of community-based intervention can be found in Tanzania, where the programme is characterized by strong community participation and management by the public sector [6]. In Tanzania, the management systems emphasized improving mothers' knowledge about growth monitoring, and using and interpreting growth charts. Integrated measures were also implemented for increasing the access of people to foodstuffs, health care services and education. This programme resulted in a higher productivity of personnel in the related sectors, when they saw how their efforts were effective in improving the nutritional status of people.

The North Karelia project in Finland is an example of a comprehensive intervention for reducing the prevalence of cardiovascular diseases [9]. Over a 25-year period, the project succeeded in decreasing the mean serum cholesterol and mortality from coronary heart disease among men aged $35-64$ years old by $18 \%$ and $73 \%$ respectively. This was achieved through cooperation between related sectors, long-term nutrition education, cooperation with volunteer organizations and food industries, promotion of food labelling policies, supporting research and international cooperation.

In the Islamic Republic of Iran a successful programme for reducing iodine deficiency disorders (IDD) was integrated into the primary health care system from 1989 [5]. Community health workers (behvarz) provided education to food retailers, members of the community and rural schoolchildren about IDD and the importance of consuming iodized salt. Consumption of iodized salt in rural areas was monitored by behvarz at the time of the annual census, through testing household salt with iodine meters. The behvarz also tested salt that children brought from their homes and contacted rural schools twice a year to identify households that did not consume iodized salt. The programme monitoring now operates at 3 levels: production, distribution and consumption. Monitoring the iodine content of urine of 8-10-year-olds is now made once a year and any required interventions are designed by inter-sectoral committees at district and province level. Integrating this programme into the primary care system and defining the duties of related sectors, including the environmental health department, food safety department, health centre laboratories, behvarz and provincial nutritionists, were important for the success of the programme. Involving different sectors inside and outside the health sector in the programme, clarifying the duties of each sector through a national committee and the existence of an effective community health network have undoubtedly contributed to the success of this IDD prevention programme.

Another participatory programme in the Islamic Republic of Iran, this time for reducing malnutrition, was performed from 1996 to 1999 in rural areas of 3 provinces [10]. It is an example of health and nutritional interventions with concomitant health and nutritional measures, including: promoting growth monitoring of children and involving mothers in using and interpreting children's growth charts; education 
about breastfeeding and complementary feeding; promoting family planning; improving sanitation and providing safe drinking water; controlling diarrhoeal diseases and education about nutrition in diarrhoea; promoting home gardening for raising vegetables; and facilitating income-generating schemes for households such as loans for livestock raising, bee keeping, carpet weaving and confectionery making. Evaluation of this programme after 3 years showed that malnutrition, according to indicators for moderate and severe underweight and stunting, had decreased by $50 \%$.

The Islamic Republic of Iran is experiencing a transition in nutritional status and so the prevalence of metabolic disorders such as obesity, cardiovascular diseases, diabetes and cancers is rapidly increasing. Thus measures have been integrated into a new national programme that includes mass media campaigns for modifying food consumption patterns and promoting physical activity, and encouraging the food industry to produce low fat, low salt and low sugar products. An evaluation of the programme is planned, to determine the effectiveness of such large-scale interventions.

Flexibility is an important factor in nutritional intervention programmes. Nutritional interventions should be tailored to each region's problems. For example, illiteracy of women may be the main reason for children's malnutrition in a rural area, so increasing women's literacy will be the priority of that region. Flexibility in managing nutritional programmes is possible only when programming and policy-making are implemented in a decentralized or regional manner. This facilitates the active involvement of the target groups in planning, implementing and evaluating nutritional interventions.

\section{Involving the community}

Participation of community and family members in nutrition improvement programmes is successful only when they take on the responsibility of health, nutrition and social welfare in the community, and acquire the necessary skills to share in the process of their own development and that of the community. Communication is vital when involving the community, because public participation is necessary for implementing interventions, from the problemdetermining phase through to offering solutions, implementation, supervision and evaluation. The community can play an important role in analysing problems and determining their own needs ("down-to-up" planning) and this has been shown to be important in the success of nutrition interventions. Community members should be informed about the problems and be encouraged to offer regional and national resources in planning, implementing and supervising nutritional programmes [11].

Different community groups, such as students, religious leaders and volunteer groups, can be involved in community nutrition improvement programmes. In the participatory programme for reducing children's malnutrition in the Islamic Republic of Iran, provincial health centres and agriculture and education ministries cooperated to facilitate and encourage schoolchildren to raise vegetables in school gardens [5]. This innovative way of promoting the consumption of vegetables and fruits aims to increasing the population's access to healthy fresh food in regions where these may not be adequately available. A further benefit is that students will transfer nutritional messages to their family members.

Another example of utilizing volunteer groups can be found in the Islamic Repub-

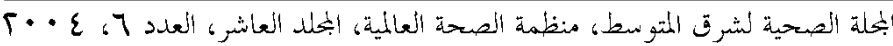


lic of Iran [2]. Women volunteers are educated about breastfeeding, complementary nutrition, nutrition of preschool children and other health-related issues by health sector personnel and nutritionists. Then each woman covers 50 households in her region for nutrition and health programmes and undertakes the necessary follow-up. A similar programme is implemented in Thailand where rural health volunteers are educated by health sector personnel and convey nutritional and health information to 10-15 rural households [7].

The best method of community participation in nutritional intervention programmes is bottom-up planning. A significant example of this can be seen in Thailand where a "basic development needs" strategy has been considered [7]. Members of the community, with the help of the public sector, gather information, identify problems, suggest solutions and implement interventions. Facilitating communities in participation in health improving programmes pays back the investment in time and resources. Community participation can be in two forms: providing some elements of the required resources or direct involvement in the decision-making process.

\section{Involving women's NGOs}

There are several successful examples of the involvement of nongovernmental organizations (NGOs) in nutritional programmes around the world. As the prime carers of the family, women have an essential role in all aspects of nutrition and women's societies can participate in educating mothers in the fields of family planning, breastfeeding, pregnancy care and nutrition of children and other vulnerable groups.
In the Islamic Republic of Iran, nutrition education and weekly iron supplementation of high-school girls, has been implemented by cooperation and financial support from a women's participation centre through the health network of the country [12]. Under the supervision of school authorities, high-school girls receive one ferrous sulfate tablet a week for 16 weeks per year and nutrition education programmes are given by teachers and school health officers.

The South-Asia Regional Office of UNICEF studied 23 community-based intervention programmes in 1995 [4]. The study showed that the success factors of the programmes included: individual and community innovations in evaluating nutritional problems; active participation of the community in identifying problems and selecting solutions; forming volunteer local committees and supporting them through the public sector; capacity building through continuous education of public sector personnel and volunteers, especially women; involving NGOs; and appropriate management and powerful leadership [4].

\section{Implementing poverty reduction programmes}

Poverty is the main factor in malnutrition and it follows that poverty reduction programmes can affect the nutrition situation of a region. Nutritional objectives should be clearly considered in these programmes, otherwise there is no guarantee that increased incomes will be spent in improving nutritional status or will support the groups that are most at-risk of malnutrition.

There is evidence of economic growth linked to a decrease in children's malnutri- 
tion in many countries [13]. In the Islamic Republic of Iran, between 1995 and 1998, underweight decreased from $16.0 \%$ to $10.5 \%$ among girls, and from $15.5 \%$ to $12.5 \%$ among boys [14]. Creating access to educational health facilities and developing structures which can mobilize the potential resources for the benefit of all, is a sustainable strategy for reducing poverty. Meanwhile, performing short- and medium-term programmes for reducing poverty in nutritional interventions is the essential condition for success of nutrition improvement programmes.

Offering food aid and supporting families with malnourished children through the cooperation of NGOs involved in food relief is one poverty reduction strategy. In Malaysia, a package of essential foods and iron and multivitamin supplements is distributed every month to poor households with malnourished children. Meanwhile, school milk programmes, which include distributing milk among primary-school students of poor rural and urban areas on specific days of the week, is another method of reducing micronutrient deficiencies among poor and vulnerable groups [15].

A similar programme is currently being carried out in the Islamic Republic of Iran. In this programme, growth-retarded children are identified through growth monitoring and screening in the primary care system and introduced to the Imam Khomeini Relief Committee, an NGO that supports poor families. A basket of food, the contents of which are defined by primary care nutritionists, is delivered free to the families of malnourished children. The experience of this programme highlights the fact that all the family members, and not only the malnourished child, need support and food aid. Inevitably, the food packages are consumed by all the family members and the effectiveness of the pro- gramme in terms of help for a particular child is reduced.

\section{Establishing and maintaining political commitment}

Political commitment is the essential prerequisite for achieving the goals of largescale nutritional programmes. To establish such a commitment, the extent and kind of problems should be recognized. Unfortunately, there are established perceptions among decision-makers and senior managers in many countries that malnutrition is simply a result of food insufficiency or that it is a medical problem, which only the health sector can deal with. The results of situation analyses should be disseminated to all levels, including the highest political positions, through seminars, workshops, publications and the mass media.

A national commitment to nutritional objectives is needed, including assigning specific resources to nutritional programmes and a willingness of government and other sectors to accept new administrative structures for tacking malnutrition, in order to facilitate the implementation of coordinated and centralized activities. Inter-sectoral coordination and mobilization for educating personnel in different sectors is an example of national commitment to achieving nutritional objectives.

Acceptance by policy-makers of a country of the importance of nutrition in health and development means that it will be considered in food regulations and support and subsidy systems. It can also lead to the establishment of a national network for identifying nutritional vulnerable groups and at-risk areas and the facilitation of inter-sectoral participation for achieving nutritional objectives. Thus establishing effective relationships with policy-makers

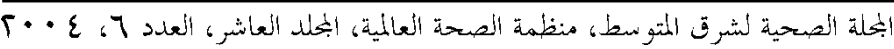


is a vital part of the successful management of nutritional programmes. So too is the establishment of food and nutrition surveillance systems, which can record and analyse positive and negative changes in nutrition measures, such as malnutrition among children and food-related metabolic disorders, and monitor the production, supply and consumption of food. Food and nutrition monitoring systems can be established in any part of the community health system. The important point is that the system should be multi-sectoral, gathering information from different sectors and disseminating it to all sectors involved. Finally, food subsidies, as a part of a government's supportive umbrella, have an important role in improving the nutritional status of communities. The effectiveness of food subsidy programmes can be increased in two ways: by targeting vulnerable groups and by ensuring that subsidised foods provide essential micronutrients as well as meeting energy requirements.

\section{Strategies for reducing micronutrient deficiencies}

\section{Supplements}

Micronutrient supplementation programmes for at-risk groups, integrated into the primary care system, are an important way to tackle nutritional deficiencies. The common deficiencies in developing countries are in iodine, iron and vitamin A. Zinc deficiency should be considered in countries that face nutritional stunting of under5-year-olds. Pregnant and lactating women and under-2-year-olds are the priority groups in supplementation programmes. If provision of supplements is possible, other at-risk groups such as the 2-5-years-olds and adolescent girls can be included. The effectiveness of providing weekly iron sup- plementation for preschool children $[16,17]$ and pregnant women $[18,19]$ has been demonstrated in many countries.

\section{Fortification of staple foods}

Fortification of staple foods with micronutrients is the main strategy for controlling micronutrient deficiencies, and through integration into the primary health care system have had useful results in the Islamic Republic of Iran. Quality control and monitoring iodized salt and fortified flour with iron and folic acid can be easily achieved by involving the related government sectors (food safety, food and drug control laboratories and environmental health) and also by educating the community and health personnel through the primary health care system. In fact, the primary health care system should be the entry point for all nutrition education programmes.

\section{Promotion of special foods}

An alternative to food fortification and supplements is the promotion of foods rich in certain vitamins or minerals. In Thailand, the Ministry of Health, with the help of the Institute of Nutrition at Mahidol University (INMU), promoted raising and consuming ivy gourd plant as a rich supply of vitamin A precursor. Using an extensive social marketing project, people were encouraged to use it in different foods. Studies in Thailand show that adopting nutrition education and food diversification strategies based on local food supplies of vitamin A, food fortification and vitamin A supplements has caused a considerable increase in serum retinol of preschool children [20].

\section{Cooperation with the food industry}

Encouraging policy-makers to support fortified food producers through assigning subsides, and acquiring the cooperation of the food industry is essential for the conti- 
nuity of fortification (nutrition) programmes.

Again, the food industry needs to be aware of a country's health objectives and be encouraged to produce more low fat, low salt and low calorie products. Paradoxically there is also evidence of an emerging problem of nutrition-related chronic diseases in developing countries, which are linked to increasing wealth and consumption of factory-produced and non-traditional foods and carbonated beverages [21]. So-called "fast foods" are believed to increase the risk of obesity and cardiovascular diseases due to their high fat content and to lead to different kinds of micronutrient deficiencies. Carbonated beverages intensify calcium deficiency, especially where there is insufficient calcium intake, due to their high phosphate content that disturbs calcium absorption.

\section{Conclusions}

Improving the nutrition of the community in developing countries requires a detailed epidemiological picture of the prevalent nutritional problems in different regions and age groups. This will make it possible to identify priorities, sensitize policy-makers, establish political commitment and design appropriate community programmes for income generation and education for the best enjoyment of food resources. Experiences acquired from community-based nutritional programmes show that ownership of a programme by the community and using a tailor-made approach are essential factors in the successful implementation of programmes [13].

The best way to increase the effectiveness of nutritional programmes is by integrating the two strategies of reducing poverty and increasing the nutritional knowledge of the community. Increasing household incomes, along with improving peoples' skills at managing household resources and adopting appropriate patterns of food consumption, increases the return on investing in the field of social development. Increasing the nutritional knowledge of the community also improves peoples' skills in managing household resources, caring for children, forming children's lifelong eating preferences and improving women's health. These in turn facilitate community participation and impact on food and nutrition policies.

\section{References}

1. Allen L, Gillespie S. What works? A review of the efficacy and effectiveness of nutrition interventions. Geneva, United Nations Administrative Committee on Coordination, Sub-Committee on Nutrition (in collaboration with the Asian Development Bank, Manila), 2001.

2. Malekafzali $\mathrm{H}$ et al. Community-based nutritional intervention for reducing malnutrition among children under 5 years of age in the Islamic Republic of Iran. Eastern Mediterranean health journal, 2000, 6(2/3):238-45.
3. Howard G, Bartram. J. Domestic water quantity, service, level and health. Geneva, World Health Organization, 2003 (WHO/SDE/WSH/03.02).

4. Nwe YY. WCARO 1995: synthesis of thematic evaluations in west and central Africa: lessons learnt from water, sanitation and guinea worm interventions. New York, United Nations Children's Fund, 1995.

5. Sheikoleslam R. Evaluation of IDD national plan in the Islamic Republic of Iran.

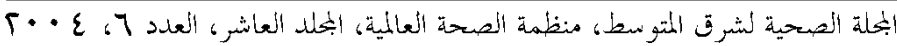


Tehran, Islamic Republic of Iran, School of Public Health, Medical University of Tehran, 1999.

6. Gillespie S, Mason J. Nutrition-relevant actions. Some experiences from the eighties and lessons for the nineties. Geneva, United Nations, 1991 (Nutrition Policy Discussion Paper No. 10).

7. Dhanamitta S. Community based nutrition projects. Institute of Nutrition, Mahidol University, Thailand, 1998.

8. Dos Santos IS, Victora C. Evaluating the efficacy of the nutritional counseling component of the "integrated management of childhood illness" strategy. Geneva, World Health Organization, 1999.

9. Puska P. Nutrition and global prevention on non-communicable diseases. Asia Pacific journal of clinical nutrition, 2002, 11(suppl.):5755-8.

10. Malekafazli $\mathrm{H}$, Sheikholeslam R. Multidisciplinary intervention model for reducing malnutrition among children in the Islamic Republic of Iran. Hakim research journal, 2003, 6(1).

11. Principles of nutrition management in primary health care. New Delhi, World Health Organization Regional Office for South-East Asia, 1995 (Regional Health Paper No. 26).

12. Samadpour $K$ et al. The effect of weekly dose of iron supplementation for 16 and 20 week on the iron status of adolescent girls in Iran. Asia Pacific journal of clinical nutrition, 2004, 13(suppl.):S135.

13. World Bank. World development report 1993. Investing in health. New York, Oxford University Press, 1993.
14. The nutritional status of children in Islamic Republic of Iran. Tehran, Islamic Republic of Iran, Ministry of Health and Medical Education, 1998.

15. Khor GL Micronutrient deficiency and its alleviation: the case of Malaysia. Asia Pacific journal of clinical nutrition, 2002, 11(suppl.):S377-81.

16. Palupi $L$ et al. Effective community intervention to improve hemoglobin status in preschoolers receiving once-weekly iron supplementation. American journal of clinical nutrition, 1997, 65:1057-61.

17. Thu BD et al. Effect of daily and weekly micronutrient supplementation on micronutrient deficiencies and growth in young Vietnamese children. American journal of clinical nutrition, 1999, 69:806.

18. Viteri FE, Ali F, Tujague J. Long term weekly iron supplementation improves and sustains non-pregnant women's iron status as well or better than currently recommended short-term daily supplementation. Journal of nutrition, 1999, 129:2013-20.

19. Tee ES et al. School-administered weekly iron-folate supplements improve hemoglobin and ferritin concentration in Malaysian adolescent girls. American journal of clinical nutrition, 1999, 69:1249-56.

20. Wasantwisut E. A combined approach to vitamin A deficiency in Thailand. Sight and life newsletter, 2002, 3:63-7.

21. Diet, nutrition and the prevention of chronic diseases: report of a joint WHO/ FAO expert consultation. Geneva, World Health Organization, 2002 (WHO Technical Report Series, No. 916). 